\title{
BESPRECHUNGSAUFSATZ
}

\section{Internationale Rohstoffpolitik und Entwicklungsländer}

\author{
von Rolf Hanisch
}

Uber den Rohstoffexport sind die Entwicklungsländer in der Kolonialzeit in den (kapitalistischen) Weltmarkt (gelegentlich erzwungenermaßen) eingebunden worden. Obwohl ein kleiner, wenn auch wachsender Kreis unter ihnen zunehmend auch als Industriewarenanbieter auf dem Weltmarkt auftritt, bestimmen die Rohstoff- und Nahrungsmittelexporte doch nach wie vor ihre Okonomien. Devisen- und Staatshaushalte, Investitionsund Konsumraten hängen in den meisten Entwicklungsländern mehr oder weniger stark von denselben ab. Viele Entwicklungsländer sind dabei nur Anbieter eines oder einiger weniger Rohprodukte ("Monokulturen"). Die Absatz- und Preisinstabilitäten, die auf vielen Rohstoffmärkten zu verzeichnen sind, schlagen daher meist voll auf sie durch und destabilisieren nicht nur ihre Okonomien, sondern über diese auch ihre politischen Systeme.

Darüber hinaus meinen die Regierungen der Entwicklungsländer - auch in langfristiger Perspektive - durch tendenziell sinkende reale Exporteinnahmen (sinkende terms-of-trade) oder allgemein durch eine "ungerechte" Bezahlung für ihre Exporte (Theorem des "ungleichen Tauschs") durch die heute noch fortwirkende, kolonial begründete internationale Arbeitsteilung in ihren Wachstumschancen blockiert zu werden. Geht man davon aus, daß der Außenhandel ein Instrument der Entwicklung ist bzw. sein könnte, so leiden die (Rohstoff-Exporte der Entwicklungsländer zudem noch unter einer sinkenden Nachfrageelastizität. Mit Ausnahme des Erdöls ist ihr Zuwachs geringer als derjenige des gesamten Welthandels, d. h. ihr relativer Anteil am Welthandel nimmt ab (These von der "Ausgliederung aus dem Welthandel").

In der Kolonialzeit verblieb den Entwicklungsländern oft nur ein Teil ihrer Rohstoffexporteinnahmen, da die Produktion - insbesondere im Bergbau und der Forstwirtschaft z. T. auch im Agrarsektor - sich in der Hand von Ausländern befand, die ihre hier anfallenden Gewinne und Einkommen retransferierten (wofür die Kolonialregime Hilfestellung leisteten). Die völkerrechtliche Unabhängigkeit bot diesen Staaten die Möglichkeit, durch Nationalisierung der Rohstoffproduktion wenigstens die Rohstoffexporteinnahmen in voller Höhe den nationalen Okonomien zugute kommen zu lassen, worum sich zahlreiche Entwicklungsländer - allerdings mit unterschiedlichem Erfolg - auch bemüh(t)en.

Die "Erringung der vollen Verfügungsgewalt über die nationalen Ressourcen" (ein Terminus aus der Diskussion um die Neue Weltwirtschaftsordnung) reicht aber faktisch ge- 
meinhin noch nicht aus, um tatsächlich über diese zu verfügen, sofern man nicht auch über die meist nötigen Input- und Produktionsmittelindustrien (den sog. "backwardlinkages") verfügt. Müssen diese noch importiert werden, geht ein guter Teil der (nun) (Brutto)Exporteinnahmen schon wieder verloren, steht also nicht dem Konsum- und dem Investitionsfonds zur Erstellung einer diversifizierten, kohärenteren Produktionsstruktur zur Verfügung. Andererseits erfolgt nur ein meist sehr kleiner Teil der Wertschöpfung eines Konsum- oder gar Investitionsgutes auf der Ebene der Rohstoffproduktion. Weitaus wichtiger ist die Verarbeitung sowie die Transport- und Vermarktungsebene (die sog. "forwardlinkages").

Seit Anfang der sechziger Jahre (Gründung der UNCTAD 1964) und verstärkt in den siebziger Jahren (seit der ersten Olkrise 1973) suchen die Entwicklungsländer diese ihre Stellung in der internationalen Arbeitsteilung durch politische Maßnahmen zu verbessern. Obwohl die genannten Problembereiche in den internationalen Verhandlungen wie auch in den unilateralen Bemühungen einzelner Entwicklungsländer eine Rolle spielen, konzentrieren die Anstrengungen sich doch auf die Stabilisierung und Erhöhung der Rohstoffexportpreise und/oder -Einnahmen. Fast alle Modelle, die hier diskutiert werden, existieren in der einen oder anderen Form schon in der Praxis. Es geht meist (nur?) darum, diesen eine breitere Verbindlichkeit zu geben und insbesondere in ihrer Arbeitsund Funktionsweise zu verbessern.

Richard Senti ${ }^{1}$ stellt in seiner Studie die bisher existierenden Organisationen zur Rohstoffexportstabilisierung vor. Internationale Warenabkommen zwischen Produzenten und Konsumenten, Produzentenvereinigungen, Konsumentenzusammenschlüsse (die Int. Energieagentur), bilaterale Abkommen zwischen den einzelnen Verbraucherländern und (u. U. einer Gruppe von) Exportländern; schließlich die Exporterlösstabilisierungsprogramme des IWF und der EG. Abschließend beschäftigt er sich mit den Reformvorschlägen der UNCTAD und entwickelt eigene Alternativen.

Obwohl Senti Okonom ist, ist er relativ sparsam in seinen Ausführungen über die Praxis der einzelnen Abkommen, die er im wesentlichen in ihren jeweiligen vertraglichen Bestimmungen vorstellt. Während er für die Internationalen Warenabkommen - in diesem Rahmen - einen ganz guten Uberblick liefert, berichtet er nur über OPEC und CIPEC als Produzentenvereinigungen (und diskutiert weitere Kartellversuche nur summarisch). Unter den bilateralen Abkommen fehlen z. B. diejenigen der Sowjetunion (etwa mit Cuba/Zucker); Abkommen über Textilien, Stahl usw. wären hingegen eher verzichtbar gewesen (da es sich bei diesen ja nicht um Rohstoffe handelt).

Abschließend stellt Senti fest, "daß die herkömmlichen Produkteabkommen sehr oft keinen oder einen nur bescheidenen Beitrag zu einem störungsfreien Welthandelt zu leisten in der Lage waren" (S. 183). Er plädiert daher für einen sehr reduzierten Anspruch an die internationalen Stabilisierungsbemühungen. Mit diesen solle man nur kurzfristige Preisfluktuationen zu bändigen trachten, man solle aber den Versuch aufgeben, langfri- 
stige Preistrends zu korrigieren versuchen. Zur Durchführung dieses Programmes schlägt er ein "Crawling-peg"-System vor, "wonach innerhalb eines bestimmten Zeitraumes die Preise der Vorperiode nur begrenzt unter- oder überschritten werden dürfen. Er gibt sich im Verlauf dieser Periode eine Abweichung vom früheren Preis, so erwächst daraus für die nächstfolgende Periode ein neuer Basispreis." (S. 196). Sollte eine derartige Preisstabilisierung gelingen, hält Senti eine Vereinheitlichung der einzelnen Abkommen für notwendig (S. 192) - was eigentlich nicht unmittelbar einleuchtet -; ferner auch eine universelle Mitgliedschaft aller wichtigen Produzenten- und Konsumentenländer, die, bekanntlich, ja nicht immer leicht zu erreichen ist, weshalb sie waus Gründen der internationalen Solidarität ... nicht mehr wie bis anhin vom einzelnen Land entschieden werden dürfen. Vielmehr sollte - analog den nationalen Gesamtarbeitsverträgen - so etwas wie die Möglichkeit der Allgemeinverbindlichkeit geschaffen werden, wonach einzelne Staaten zur Mitarbeit und Einhaltung der Abkommen verpflichtet sind, wenn eine im voraus bestimmte Mehrheit von Ländern sich in diesem Sinne entscheidet" (S. 193). Außerdem tritt er für die Unbefristetheit und Nichtkündbarkeit der Abkommen, sowie für Liefer- und Abnahmeverpflichtungen innerhalb bestimmter Preisgrenzen ein.

Obwohl die eigentlichen Zielsetzungen des Stabilisierungsmodells von Senti eher moderat sind, dürften seine Durchführungspostulate noch viel weniger durchzusetzen sein, als alle anderen in der Diskussion befindlichen Modelle, da sie ja hier einen erheblichen Souveränitätsverlust praktisch aller Nationalstaaten implizieren. Ob sie überhaupt darüberhinaus praktisch durchführbar wären und einen ökonomischen Sinn ergäben (wie etwa die Liefer- und Abnahmeverpflichtungen), muß gleichfalls sehr bezweifelt werden.

Während Senti den Inhalt der Warenabkommen nur zusammenfassend vermittelt (was einer schnelleren Orientierung dienlich ist), finden sich in dem Band der beiden Göttinger Völkerrechtler Schirmer und Meyer-Wöbse² die vollen Vertragstexte der gegenwärtig geltenden Warenabkommen sowie Auszüge aus früheren Warenabkommen, einigen Produzentenabkommen und weiteren internationalen Dokumenten zur Rohstoffpolitik. Die Dokumente werden ergänzt durch eine Zusammenstellung wesentlicher Vertragsdaten (inkl. den Adressen der meisten Organisationen), einer (allerdings etwas willkürlich zusammengestellten) Auswahlbibliographie und einer hervorragenden Einleitung (135 S.) zur Geschichte und Diplomatie der internationalen Rohstoffpolitik, die mit völkerrechtlicher Akzentuierung geschrieben ist, gleichwohl die reale Praxis angemessen berücksichtigt und bestens zur Einführung in das Studium der internationalen Rohstoffpolitik geeignet ist. Für Völkerrechtler, aber auch Politologen und Ökonomen, die sich mit Rohstofffragen beschäftigen, ist dieser Band damit ein sehr nützliches und unentbehrliches Hilfsmittel. Als hauptsächliches Defizit dieses (allerdings schon so voluminösen) Buches muß nur erwähnt werden, daß die Vertragsdokumente nur weniger Produzentenvereinigungen berücksichtigt werden. Diese sind allerdings auch wesentlich schwerer zugänglich (nichtsdestoweniger aber dennoch erhältlich) als die Texte der Interna-

2 Wilhelm G. Schirmer/Gerhard Meyer-Wöbse: Internationale Rohstoffabkommen. Vertragstexte mit einer Einführung und Bibliographie. München, New York, London, Paris: K. G. Saur 1980, 907 S., 150 DM. 
tionalen Warenabkommen, weshalb eine Hilfestellung durch diese Dokumentation besonders willkommen gewesen wäre.

Der von Theodor Dams und Gerhard Grohs ${ }^{3}$ herausgegebene Sammelband vereinigt zwölf Referate einer Konferenz, die unter der Federführung der Wissenschaftlichen Kommission des Katholischen Arbeitskreises Entwicklung und Frieden (KAEF), mit 60 Experten aus Wissenschaft, Wirtschaft, Verwaltung und Politik im April 1977 in Bonn stattgefunden hat sowie eine (durchaus sehr lesenswerte) Auswahl der Diskussionsprotokolle derselben. Wer einen Uberblick über die verschiedenen Positionen gewinnen will, die in der internationalen Rohstoffpolitik vertreten werden, für den ist dieser Band eine nützliche Lektüre.

Die Referate und die Diskussion wurden in zwei Abschnitte gegliedert. Im ersten Teil werden die Grundlagen der gegenwärtigen rohstoffpolitischen Diskussion, im zweiten Instrumente der Rohstoffpolitik, insbesondere der Gemeinsame Fond der UNCTAD, Stabex der EG u. a. Modelle, behandelt. Im Rahmen dieses Besprechungsaufsatzes ist es nicht möglich, auf alle Positionen in diesem Band einzugehen, zumal es sich überwiegend nicht um ganz neue Gedanken handelt, die hier vorgetragen wurden (womit im vorliegenden Fall durchaus keine negative Aussage gemacht werden soll). Einige Argumente seien aber doch erwähnt. So setzt sich Lütz Hoffmann, Volkswirt in Regensburg, mit den Ursachen und zukünftigen Aussichten der Rohstoffpreisentwicklung auseinander. Er hält es u. a. für recht unrealistisch, die Rohstoffpreise durch Kartellmaßnahmen erheblich zu steigern und für die Entwicklung nutzbar zu machen (S. 33 ff.). Selbst wenn Preissteigerungen durchsetzbar seien, hätten diese zur Konsequenz, daß der Aufbau von Industriebetrieben - im Verhältnis zu Investitionen in der Rohstoffexportproduktion relativ an Attraktivität verlieren würde und auch die Absatzchancen in den Industrieländern abnehmen würden. Damit würde sich der Strukturwandel in den Entwicklungs- wie den Industrieländern verlangsamen und das Wachstumstempo abschwächen. Langfristig würde eine Erhöhung der Rohstoffpreise zu einer Ausweitung der Produktion und, ohne entsprechende Steigerung der Nachfrage, zu schließlich doch wieder sinkenden Preisen bzw. Preisverhältnissen (zu den Fertigwaren) führen. Am Ende würden die Rohstoffländer schlechter dastehen als zuvor. Dieser Kreislauf könnte nur durchbrochen werden, wenn auch das Rohstoffangebot wirksam kontrolliert und eingeschränkt würde. Allerdings sei dazu allein der Arbeitsmarktspielraum allgemein sehr schwach, selbst wenn man radikal arbeitsintensive Produktionszweige der Industrie fördern würde. Hoffmann hält daher nur Bemühungen, kurzfristige Preisschwankungen auszugleichen für realistisch und sinnvoll. Hierzu könnten auch Kartelle einen sinnvollen Beitrag leisten. Allerdings würde eine optimale Preis- und Einkommensentwicklung kaum denkbar sein, wenn man von quasi identischen Produzenten- und Verbraucherpreisen ausgeht, da beide ganz verschiedene Funktionen zu erfüllen hätten (der Produzentenpreis darf nicht

3 Theodor Dams/Gerhard Grohs (Hrsg.): Kontroversen in der internationalen Rohstoffpolitik. Ein Beitrag zur Rohstoffpolitik der Bundesrepublik Deutschland nach UNCTAD V, München, Mainz: Kaiser, Grünewald $1977,255 \mathrm{~S}$. 
Uberproduktion induzieren, der Verbraucherpreis sollte nicht die Nachfrage zurückgehen lassen, (bei nicht vermehrbaren Rohstoffen) aber auch nur mit geringer Rate steigen lassen). Hoffmann bereichert die Diskussion durch den Vorschlag, daß die Differenz von den Industrieländern durch variable Importzölle abgeschöpft und den Entwicklungsländern zum Zwecke der Umstrukturierung ihrer Wirtschaften zur Verfügung gestellt werden sollten. Wenn man etwa daran denkt, daß die Steuern auf Kaffee und Erdöl (in der Bundesrepublik) etwa auch den Exportpreisen der Rohstoffproduzenten in voller Höhe entsprechen, so würde es hier eigentlich "nur" um die Ubertragung dieser Mittel an die Entwicklungsländer gehen, wofür ein Verteilungsschlüssel auszuhandeln wäre. An einen derartigen Automatismus sind die Industrieländer natürlich nicht interessiert, weshalb dieses Konzept kaum größere politische Chancen der Verwirklichung besitzt als alle anderen in der Diskussion weitgehend festgefahrenen Modelle.

Immerhin würde ein Problem dadurch weitgehend ausgeräumt werden, worauf die Mitarbeiter des Kieler Instituts für Weltwirtschaft, hier vertreten durch Bernd Stecher, immer wieder hinweisen: die Unmöglichkeit durch Rohstoffabkommen bzw. sogar durch Indexierungsversuche die Gleichgewichtspreise, die in einem marktwirtschaftlichen System durch Angebot und Nachfrage gefunden werden, richtig zu prognostizieren (S. 45). Gelänge dies aber nicht, wären auf globaler Ebene ebensolche Uberschüsse zu erwarten, wie sie auf dem EG-Agrarmarkt auch schon üblich sind und auch dort schon immer unlösbarere Probleme aufwerfen.

Ausführlicher kann man die "Kieler Position" in dem von Bernd Stecher zusammen mit Stefan Baron und Hans. H. Glismann ${ }^{4}$ verfaßten Studie nachlesen, die auf einen Gutachterauftrag des Bundesministeriums für Wirtschaft zurückgeht. Die Autoren machen hier noch einmal ihre Bedenken gegen die Operationalisierbarkeit von internationalen Regulierungsversuchen deutlich. Sie weisen insbesondere auf den Umstand hin, daß durch eine Stabilisierung der Rohstoffpreise eine Erlösstabilisierung nur dort denkbar ist, wo die Preise in erster Linie nachfragebedingt schwanken (nach den Autoren nur bei Kautschuk, Eisenerz, Tee, Kupfer, Aluminium). In allen anderen Fällen würde eine Stabilisierung der Rohstoffpreise nur die bestehenden Schwankungen der Exporterlöse erhöhen (S. 25).

In einem zweiten Schritt untersuchen sie (ökonometrisch) welche Wohlfahrtseffekte eine Erhöhung der Rohstoffpreise um 10 \% über dem Gleichgewichtspreis (der einen Ausgleich von Angebot und Nachfrage garantiert) haben würde. Nach ihren Berechnungen würden bei den 10 Haupt-Rohstoffen ("Core Commodities") den Exportländern eine Produzentenrente von $+10,4$ Mrd. $\$$ zufließen. Die Importländer hätten eine zusätzliche Konsumentenrente von 10,1 Mrd. \$ zu bezahlen und es würden Vernichtungskosten (der Uberschüsse, aufgrund steigender Produktion und sinkender Nachfrage) über -8 Mrd. \$ anfallen. Mithin wäre der weltwirtschaftliche Wohlfahrtsverlust, den die Importländer zu tragen hätten (bei Teilung der Vernichtungskosten: Importländer - 14,1

4 Stefan Baron/Hans H. Glismann/Bernd Stecher: Internationale Rohstoffpolitik. Ziele, Mittel, Kosten, Tübingen: J. C. B. Mohr (Paul Siebeck) 1977, 194 S., 28 DM. 
Mrd. \$, Exportländer $+6,4$ Mrd. \$), erheblich höher als der Nutzen, den die Entwicklungsländer daraus ziehen würden. Es wäre daher, nach Ansicht der Autoren, für alle Seiten ertragreicher, wenn die Konsumenten zwischen 14,1 - 6,4 Mrd. $\$$ an die Produzenten direkt überweisen würden, "um diese zu bewegen, auf die Durchsetzung 'gerechter Preiser zu verzichten" (S. 35). Allerdings gäbe es auch in diesem Fall die bekannten Allokationsprobleme, da allein 11 Exportländer (darunter nur sieben Entwicklungsländer, dazu Australien, Frankreich, USA, SU) etwa die Hälfte der Gesamtbegünstigung auf sich vereinigen würden (S. 43).

Preissteigerungen, die Rohstoffkartelle in der Lage sind durchzusetzen, sind hingegen rechnerisch billiger, da Rohstoffkartelle nur funktionieren würden, wenn sie eine Uberschußproduktion vermeiden könnten, mithin Vernichtungskosten nicht anfallen würden. Genau hier liegt aber auch der springende Punkt, der über die Viabilität von Kartellen entscheidet.

Die hier vorgelegten Berechnungen gehen von der Annahme aus, daß es sich bei den gegenwärtigen Rohstoffpreisen um Markt- und Gleichgewichtspreise handelt, die durch Rohstoffabkommen administrativ verschoben werden sollen. Tatsächlich trifft diese Annahme aber nur auf einige Güter (etwa tropische Produkte und einige andere Agrargüter), auf andere aber nicht zu. So ist die Preisgestaltung auf dem "freien" Zuckerweltmarkt nicht verständlich, wenn man die politischen Ungleichgewichtspreise in einigen großen Produktions- und Verbraucherländern nicht auch berücksichtigt, woraus sich die besonderen Schwierigkeiten für internationale Regulierungsbemühungen ergeben, wenn diese Märkte nicht einbezogen werden (können). Gravierender noch ist, daß die Differentialrentenfrage - relevant vor allem bei der Produktion nicht-erneuerbarer Rohstoffe - hier völlig ausgeblendet wird; d. h. es wird der Umstand nicht thematisiert, daß aufgrund der (häufig) billigeren Produktionskosten in den Entwicklungsländern, eine Erhöhung der Rohstoffpreise bzw. des Förderzinses nicht unbedingt zu höheren Marktpreisen führen muß bzw. allenfalls die Extragewinne der TNK (die diese Differentialrenten bisher eingestrichen haben) mindern würde.

Auch die anderen Projekte, die auf dem "Nord-Süd "Tisch liegen, Indexierung der Rohstoff- an die Industriewarenpreise sowie Exporterlösstabilisierung über eine kompensatorische Finanzierung, halten Baron et. alii für kaum durchführbar bzw. nicht ziel-mittelkompatibel. So weisen sie u. a. auf die Schwierigkeiten hin, die sowohl bei einer länderspezifischen als auch güterspezifischen Indexierung auftreten würden: Im ersten Fall würden dieselben Rohstoffe verschiedene Exportpreisanhebungen erfahren, da sie an unterschiedliche Importwarenkörbe gekoppelt wären; im zweiten Fall wäre die Wirkung für die einzelnen Entwicklungsländer wieder sehr unterschiedlich, wenn nicht alle Rohstoffe indexiert würden. Damit würden aber wiederum die rohstoffexportierenden Industrieländer mehr begünstigt als eben die Entwicklungsländer (da diese wertmäßig mehr Rohstoffe (ohne Öl) als die letzteren exportieren würden), andererseits müßten viele (andere) Entwicklungsländer, inzwischen auch nicht unbedeutende Rohrstoffimporteure, die erhöhten Kosten mittragen (S. 65 ff.). Eine kompensatorische Finanzierung schließlich hätte nach Ansicht der Autoren nur einen Sinn, wenn der Transfer vom Empfänger 
zu marktüblichen Bedingungen zu verzinsen ist, da andernfalls eine reine Entwicklungshilfe administrativ leichter (und damit billiger) erfolgen könnte (S. 81).

Als Alternative zu diesem "dirigistischen Ansatz der UNCTAD" empfehlen die Kieler Autoren eine "im Kern marktwirtschaftliche Konzeption", im Rahmen derer die Entwicklungsländer ihren Wachstumsspielraum in der Importsubstitution, bei der Diversifizierung ihres Exportangebots, bei der in mittlerer und längerer Sicht Halb- und Fertigwaren die Rohstoffe zu ersetzen hätten, nutzen sollten. Im Rahmen dieser Strategie sollte eine Liberalisierung des internationalen Ressourcentransfers (Direktinvestitionen!) ermöglicht, eine Unterbewertung der Währungen der Entwicklungsländer akzeptiert und die Handelshemmnisse in den Industrieländern abgebaut werden.

Dieser Vorschlag hat aus bundesrepublikanischer Sicht den Vorteil, daß er die Entwicklungsländer in erster Linie auf ihre eigenen Möglichkeiten und ihre Eigenanstrengungen verweist. Auch die dazu nötige Offnung unserer Märkte für Halb- und Fertigwarenexporte aus den Entwicklungsländern liegt noch durchaus auf der verbal proklamierten Linie der Regierung in Bonn. Allerdings war man bisher, immer wenn es konkret wurde (siehe z. B. Textilimporte und das Welttextilabkommen zur Importdrosselung; auch ein administrativ regulierter Markt!), nicht bereit, auch selbst Konsequenzen aus dieser "marktwirtschaftlichen Entwicklungsstrategie" zu ziehen. Tatsächlich hat die Bundesregierung wohl auch kaum eine eigene Konzeption für den Nord-Süd-Dialog und zur Lösung der weltwirtschaftlichen Globalprobleme. Die bisherige Politik war allein durch ein hinhaltendes Taktieren (möglichst im Schatten der USA) bestimmt, letztlich mit dem Ziel, an der bestehenden Weltwirtschaftsordnung (inklusive den auch von uns verschuldeten "marktwirtschaftlichen" Ungereimtheiten) nichts oder nichts Grundsätzliches zu ändern und ändern zu lassen. Das Gutachten der Kieler Okonomen dürfte diesen fatalen Immobilismus wohl eher noch weiter fördern, da es eine Reihe guter oder doch (zunächst) plausibler Argumente gegen die UNCTAD-Position zusammenträgt. Es ist aber mehr als fraglich, ob die hier auch aufgezeigte, allerdings wesentlich knapper formulierte, konstruktive Alternative zum Abbau der Konzeptionslosigkeit in der praktischen Politik tatsächlich beiträgt. Es hat eher den Anschein, daß die Bundesregierung ihren destruktiven hinhaltenden Widerstand gegen die UNCTAD-Position nicht durch eine konstruktive offensive Strategie, etwa im Sinne der Kieler Position (die ja durchaus auch schon zum rhetorischen Gemeingut der Politiker gehört), ersetzt. Politisch zu einer Entscheidung gezwungen ist man dann doch eher bereit einige (kleine) Schritte in Richtung UNCTAD-Dirigismus zu gehen (siehe Gemeinsame Fond!), als daß man die Konjunktur- und Strukturkrise im eigenen Land noch zusätzlich durch einen dann notwendig werdenden verstärkten Strukturwandel noch weiter zu belasten bereit wäre.

Seit den (unilateralen) Aktionen der Erdölländer wird auch verstärkt diskutiert, ob die rohstoffproduzierenden Entwicklungsländer selbst durch eigene Kraft ihre Interessen auf den Rohstoffweltmärkten durchsetzen können. Im Rampenlicht stehen hier die Erdölexportländer und ihre Organisation, die OPEC, über die mittlerweile zahlreiche Veröffentlichungen von Journalisten, Wissenschaftlern und Beteiligten (ehemaligen Generalsekretären der OPEC bzw. Erdölminister) vorliegen. 
Eine der verläßlichsten und informativsten Studien über die OPEC hat kürzlich Ian Seymor $^{5}$ vorgelegt, der als Mitarbeiter des renommierten und für die Tagesinformation unerläßlichen Middle East Economic Survey (aus Zypern) seit Jahrzehnten den Erdölweltmarkt beobachtet und dabei auch Gelegenheit hatte, mit vielen beteiligten Akteuren zu sprechen.

Seymor berichtet im Hauptteil seines Buches chronologisch in acht Kapiteln über die Entwicklung der OPEC und die (individuellen) Aktionen der Olexportstaaten (seit 1960) zur Ausweitung ihres Anteils an der Bondenrente sowie zur Kontrolle der Preise. Er macht deutlich, daß auch in den sechziger Jahren die Olländer nicht unbeachtliche Erfolge schon erzielen konnten, daß ihnen der große Durchbruch in der Rentenfrage 1971, in der Preisfrage 1973 gelang, daß sie 1975-78 eine Erosion der realen Preise nicht aufzuhalten vermochten und erst die Ereignisse im Zusammenhang mit der Iranischen Krise (1979) eine zweite Preisexplosion möglich machten. Uber all diese Vorgänge berichtet er sehr detailliert und abgewogen und macht die verschiedenen Positionen der einzelnen Olländer sowie die Rolle der OPEC deutlich. In einem abschließenden zweiten (kürzeren) Teil geht er noch auf einige Sachfragen ein, nämlich auf die Frage der (vergeblichen Bemühungen zur) Produktionsregulierung, der Managementkontrolle, der Entwicklungshilfe der OPEC und schließlich deren Stellung im Nord-Süd-Dialog. Wer immer sich über die Olpolitik der letzten beiden Jahrzehnte oder auch über die internationalen Beziehungen des Mittleren Ostens und Nordafrikas informieren will, sollte die Studie von Seymor nicht übersehen.

Im Gegensatz dazu bietet die Arbeit des Irakers Al-Chalabi6 eigentlich "nichts Neues". Obwohl man sich aufgrund der Vita des Autors (eines führenden Olpolitikers seines Landes, 1976-78 stellvertretender Generalsekretär der OAPEC, seither bei der OPEC) auch Informationen über einige Interna oder bisher unbekannte Zusammenhänge erhoffen mag, wird man hier weitgehend enttäuscht werden. Auch eröffnet die Studie kaum neue Erkenntnisse, wenn man sie unter dem Blickwinkel "Selbstverständnis der OPEC (bzw. OAPEC)" betrachtet, obwohl sie dafür eigentlich geeigneter sein könnte, als die aus dieser Sicht in dieser Zeitschrift schọn rezensierte Magisterarbeit eines (späteren) Erdölministers. ${ }^{7}$ Immerhin handelt es sich aber auch hier um eine durchaus brauchbare knappe Einführung in die Olpolitik und die Rolle der OPEC, die aber mit einigen vergleichbaren Veröffentlichungen auf ähnlichem Niveau konkurriert.

Im Gegensatz zur OPEC ist die 1968 gegründete Organization of Arab Petroleum Exporting Countries (OAPEC) weit seltener Gegenstand der wissenschaftlichen Darstellung und Erörterung. Auch in den beiden eben vorgestellten Monographien wird diese Organisation allenfalls am Rande erwähnt. Diese Lücke schließt die Arbeit des Ägypters

5 Ian Seymor: OPEC. Instrument of change. London: Macmillan 1980, 306 S.

6 Fadhil J. Al-Chalabi: OPEC and the international oil industry: A changing structure. Oxford: University Press $1980,165 \mathrm{~S}$.

7 VRU 3/1979, S. $259 \mathrm{ff}$. 
El-Gebali, ${ }^{8}$ die in Kiel als juristische Dissertation angenommen wurde. Der Autor beschäftigt sich nach einer knappen Einführung in die Vorgeschichte mit den Rechtsfragen der Mitgliedschaft, den verschiedenen Organen der OAPEC, den Aktionen und Handlungen der OAPEC, schließlich ihren Beziehungen zu anderen internationalen Organisationen. Für den Juristen El-Gebali steht notwendigerweise die (auch kategoriale) Erörterung der Rechtsfragen und die Darstellung der Normen der Organisation (Satzung usw.) im Vordergrund. Da er aber auch genügend Informationen über das tatsächliche Funktionieren der OAPEC, also über deren "Rechtspraxis", aufbereitet, ist diese Studie auch von Sozialwissenschaftlern mit Gewinn zu lesen.

Allerdings wird man dem Autor nicht in allen Fragen folgen können. So widmet er der Suspensierung Ägyptens aus der OAPEC (im April 1979) breiten Raum. Diese war durch keine Satzungsregelung gedeckt; El-Gebali bezweifelt auch ihre Rechtmäßigkeit. Als Grund für seine Auffassung führt er im wesentlichen an, daß es bisher nur sehr wenige Ausschlüsse von Mitgliedern aus internationalen Organisationen ohne Satzungsermächtigung gegeben habe und in diesen Fällen auch die Rechtsüberzeugung der beteiligten Akteure gefehlt habe. Obwohl in der Satzung der OAPEC auf mögliche politische Zielsetzungen dieser Organisation nicht bezug genommen wird, sondern nur von einer Vereinheitlichung der Erdölpolitik usw. gesprochen wird, haben Gründer und (spätere) Mitglieder mit ihr auch immer eine politische Zielsetzung ("Kampf gegen Israel“) verbunden, worauf El-Gebali mit mehreren Belegen hinweist (S. 34 ff.). Einen in diesem Sinne möglichen Vertragsbruch Ägyptens mit seiner "Camp-David-Politik" (etwa im Sinne der Wiener-Vertrags-Konvention) lehnt er jedoch gleichfalls ab, da auch durch diese Politik besetztes arabisches Land wieder befreit worden sei (was ein Vertragsziel sei). Bei aller Sympathie für die sachliche Position des Akteurs Ägypten, bleibt aber eigentlich nicht einzusehen, daß die Mitgliedschaft eines Landes in einer internationalen Organisation nicht suspensiert werden könne, wenn alle anderen Mitglieder dieser Organisation der Meinung sind, daß dasselbe sich nicht vertragskonform verhält und eine weitere Zusammenarbeit für nicht zumutbar halten. Wie gesagt, hiermit soll kein Urteil über die politische Kontroverse selbst gefällt werden. In diesem Zusammenhang trägt der Umstand übrigens nicht gerade zur Klärung bei, daß 1973 die "Olwaffe" von der Ólministerkonferenz der OAPEC-Staaten beschlossen wurde, die OAPEC aber selbst eine eigene Beteiligung daran in Abrede stellte, um ihren rein wirtschaftlichen Charakter zu betonen (S. $138 \mathrm{ff}$.).

Die Embargo-Maßnahmen wurden so also von einer Konferenz der arabischen Erdölminister, einem "eigens dafür zusammengetretenen politischen Gremium", beschlossen, das personell mit dem höchsten Organ der OAPEC identisch war. Die OAPEC führte dann allerdings die Beschlüsse dieses Gremiums aus und übernahm damit allein die Koordination der einzelnen Maßnahmen, "die von ihr als technische Aufgabe der Si-

8 Salah El-Gebali: Die OAPEC als Organisation zur Interessenvertretung der Arabischen Olländer in den internationalen wirtschaftlichen Beziehungen. Frankfurt a. M., Bern: Peter D. Lang 1981, 255 S. 
cherstellung der Einheitlichkeit im Olexport, losgelöst von dem politischen Hintergrund, betrachtet werden konnte" (S. 141).

Die Ölpreisrevolutionen, die die OAPEC- und OPEC-Länder 1973 und 1979 initiierten bzw. durchsetzen konnten, trafen nicht nur die Hauptverbraucherländer - die westlichen Industrieländer - sondern natürlich auch die anderen ölimportierenden Entwicklungsländer. Diese Beziehungen zwischen OPEC-Ländern und den übrigen Entwicklungsländern ("NOPEC॥) wird in einer jüngst erschienenen Monographie von Paul Hallwood und Stuart Sinclair ${ }^{9}$ untersucht. In dieser sehr sorgfältig und materialreich argumentierenden Studie treten die Autoren der Ansicht entgegen, daß die allgemeine Wirtschaftskrise der 70er Jahre allein durch die Erdölpreiserhöhungen verursacht worden sei. Vielmehr seien dafür eine Reihe anderer Faktoren gleichfalls verantwortlich (zyklische Kondratieff-Rezension, Weltwährungskrise, Inflationmasender Protektionismus usw.). Auch hätten die Olpreissteigerungen generell zwar zu einer erheblichen Verschlechteriung der terms-of-trade aller "NOPEC"-Länder geführt, die Verschärfung der Zahlungsbilanzkrisen und des Verschuldungsproblems einiger Entwicklungsländer kann aber gleichfalls nur z. T. auf sie zurückgeführt werden. Tatsächlich haben einige Entwicklungsländer am "Oilbonanza"durch den Empfang arabischer Entwicklungshilfe auch partizipiert, die allerdings vornehmlich unter politischen Gesichtspunkten verteilt wird, d. h. zunächst an die sog. "Frontstaaten" (gegen Israel), dann an die anderen arabischen sowie islamischen und afrikanischen Staaten und Indien (und erst in einer zweiten Welle etwas breiter gestreut wurden). Von der erstgenannten Gruppe abgesehen, lag die überproportional gesteigerte Olrechnung jedoch über diesen nicht-kommerziellen Zuwendungen, die überwiegend von einigen bevölkerungsarmen Olländern getragen werden. Es genügt in diesem Zusammenhang natürlich nicht, nur die arabische Entwicklungshilfe mit der höheren Olrechnung zu vergleichen. Hallwood/Sinclair machen auch deutlich, daß am Importboom der Olländer die anderen Entwicklungsländer durch überproportionale Steigerung ihrer Exporte und durch die Entsendung von Arbeitsmigranten in diesen Raum ganz erheblich profitieren. (wobei sie allerdings nicht versäumen, den durchaus zweischneidigen Charakter der Arbeitsmigration herauszuarbeiten). Auch hier profitiert allerdings wiederum nur ein kleiner Kreis (süd- und ostasiatischer) Entwicklungsländer, während alle übrigen daran kaum beteiligt werden. So untermauert die Studie den Schluß, daß die Olpreissteigerungen den Differenzierungsprozeß zwischen den Entwicklungsländern - der allerdings schon in den sechziger Jahren eingesetzt hatte - noch weiter verschärft und akzentuiert hat. Sie wendet sich allerdings gegen eine Úberdramatisierung der Rolle der Olländer in diesem Zusammenhang.

Damit bleibt die Frage, ob Entwicklungsländer auf anderen Rohstoffmärkten nicht ähnliche Erfolge erzielen könnten. Oder: Was ist eigentlich die Ursache für den Erfolg der Olländer? Hallwood/Sinclair sehen ihn in erster Linie in der gegenwärtig relativ inelastischen Nachfrage (und man könnte hinzufügen: in den hohen Differentialrenten in den

9 Paul Hallwood/Stuart W. Sinclair: Oil, debt and development: OPEC in the Third World. London: Georg Allen \& Unwin 1981, $206 \mathrm{~S}$. 
Entwicklungsländern) begründet. Der OPEC selbst messen sie nur eine geringe Bedeutung bei. Sie sei als internationale Organisation schwach und von den Mitgliedern auch bewußt schwach gehalten, "stark" seien vielmehr nur die einzelnen Mitgliedsländer, die - wiewohl in enger Kommunikation untereinander stehend - die meisten Entscheidungen über Olpreise, Förderungspolitik, Steuer und Royalties, Verstaatlichungen individuell treffen bzw. getroffen haben. Eine kritische Rolle spielen hier insbesondere die bevölkerungsarmen Exportländer als "Residual-Versorger", die also, ohne negative Konsequenzen für ihre Entwicklungspläne usw., ihre Produktion gegebenenfalls einschränken und ausweiten können. Diesen Ländern (insbesondere am Golf) wurde so schon die Eigenschaft eines Sub-Kartells zugesprochen, obwohl es auch Beispiele dafür gibt, daß diese nicht so funktioniert haben (S. 50). Immerhin sehen Hallwood/Sinclair in dem Umstand, daß eine Produktionskontrolle der OPEC-Länder nicht nötig war, um die Preise zu bestimmen, eine Ursache für ihre Stärke, da dadurch entsprechendes Gezänk auch vermieden werden konnte (S. 48). Wie sieht es aber nun mit den anderen Rohstoffen aus?

Die Literatur über Kartellmöglichkeiten und -Perspektiven sowie Produzentenvereinigungen für alle anderen (mineralischen und agrarischen) Rohstoffmärkte ist weit spärlicher als über Erdöl und die OPEC. Wie die erfolgreichen Aktionen der Olländer 1973 zu einem gewissen Nachahmungs-Gründungsboom von Produzentenvereinigungen geführt haben, so wird immerhin seither auch dieser Frage - und zwar sowohl unter dem Blickwinkel der Bedrohung der westlichen Industrieländer wie auch der Emanzipation der Entwicklungsländer - etwas größere Aufmerksamkeit beigemessen. Obwohl es mittlerweile (außer OPEC und OAPEC) 15 solcher Produzentenvereinigungen von Entwicklungsländern gibt, deren älteste schon 1960 gegründet wurden, gibt es nach wie vor sehr wenige empirische Studien über dieselben. Es überwiegen kategoriale Diskussionen, in denen auf allgemeiner Ebene die möglichen Perspektiven von Produzentenaktionen diskutiert werden, ohne daß man sich deren tatsächlichen Aktionen bzw. die diese aktuell behindernden Schwierigkeiten und Probleme näher ansieht.

In diese Kategorie fällt voll die von der DGFK geförderte Studie von Manfred Tietzel, ${ }^{10}$ die sich - neben den Rohstoffkartellmöglichkeiten - auch noch mit den Rohstoffabkommen und der Absorptionsfähigkeit der Olländer Iran und Saudi-Arabien beschäftigt (ohne aber OPEC bzw. OAPEC zu behandeln). In diesem überwiegend redundant geschriebenen Buch, nicht frei von größeren und kleineren Fehlern und Auslassungen, diskutiert der Autor, in dem hier interessierenden Teil, zunächst die Faktoren, die seiner Ansicht nach die Kartellfähigkeit begünstigen: Homogenität und Lagerfähigkeit der Rohstoffe, potentielle Substitutionskonkurrenz, Monopolisierungsgrad des Angebots, Verhältnis der Exportabhängigkeit zur Weltmarktstellung auf einem Rohstoffsektor, die allgemeinen Währungsreserven, schließlich die Elastizität des Angebots, der Preise und der Nachfrageeinkommen. Tietzel untersucht dann in einem zweiten Schritt neun

10 Manfred Tietzel: Internationale Rohstoffpolitik. Eine Analyse der rohstoffpolitischen Aspekte des NordSüd-Dialogs. Bonn-Bad Godesberg: Verlag Neue Gesellschaft 1977, 156 S. 
Rohstoffe - Kupfer, Kaffee, Zucker, Baumwolle, Kautschuk, Zinn, Bauxit, Phosphat, Blei - bemerkenswerterweise nicht Kakao, wo eine der markantesten Kartellaktionen stattfand! - ohne allerdings seine oben als wichtig charakterisierten Faktoren hier immer am Beispiel der einzelnen Rohstoffe auszudiskutieren. Grosso modo sieht er die Wahrscheinlichkeit einer (erfolgreichen) Kartellbildung auf den von ihm behandelten Rohstoffmärkten für gering an, wenn auch aus jeweils unterschiedlichen Gründen. Auch vom Ol hat man dies allerdings in den 50er und 60er Jahren (und noch Anfang der 70er Jahre) gesagt. Erscheint diese allgemeine Schlußfolgerung, unter den gegebenen Bedingungen, plausibel und einsichtig, so bietet uns das beliebige Aneinanderreihen von verschiedenen Indikatoren, ohne daß offenbar etwas über deren Wertigkeit und Beziehungsmuster untereinander gesagt werden könnte, noch kein allzu brauchbares prognostisches Instrumentarium. Hier wäre, wie gesagt, eine genauere Kenntnis der real stattfindenden Handlungen und ihrer Analyse im gegenwärtigen Stadium wohl noch sinnvoller. Zu dieser Kärnerarbeit, die eben nicht allein aus einer Bibliothek geleistet werden kann, sind allerdings allzu viele Kollegen noch nicht bereit gewesen.

Auch die Studie des norwegischen Friedensforschers Helge Hveem ${ }^{11}$ ist auf diesem Gebiet zwar ergiebiger als die von Tietzel, vollauf befriedigen kann sie aber auch nicht. Sie ist der Tietzel'schen Arbeit an Aufbau, Originalität und Materialreichtum allerdings überlegen. Hveem sieht die Rohstoffmärkte durch nicht-marktkonforme Eingriffe und "Marktmacht" manipuliert, nämlich durch die Fähigkeit und die Neigung von einzelnen Teilnehmern, den Markt zu ihrem eigenen Vorteil, zur Durchsetzung politischer, militärisch-strategischer und spezifischer ökonomischer Interessen, zu beeinflussen (S. 13). Das imperfekte Funktionieren des Marktes sei so eher die Regel, denn die Ausnahme, und dies geschehe allgemein auf Kosten der Entwicklungsländer. Aus diesem Grunde habe auch die "Rohstoffmacht" (conmodity power) der Entwicklungsländer organisiert zu werden.

Hveem untersucht so zunächst die einzelnen Faktoren der Macht auf den Rohstoffmärkten (geogr. Konzentration, Organisation, Finanzen, Information, Technologie, Rolle des Staates) und der Verteilung der Nutznießung aus der Rohstoffexportproduktion. In einem zweiten Abschnitt stellt er verschiedene Typen von Produzentenabkommen den internationalen Warenabkommen und andere Rohstoffe betreffenden Vereinbarungen gegenüber. In seinem Hauptteil wendet er sich schließlich den Bedingungen und Grenzen für die Etablierung von Produzentenvereinigungen zu (untergliedert in politische, ökonomische, technologische, ökologische Faktoren), um sich abschließend mit der Rationalität von Kollektivaktionen und der kollektiven 'self-reliance', Strategiefragen, den Implikationen für die Produktions- und Vermarktungspolitik und möglichen Organisationsfragen im Rahmen der 'Gruppe der 77, zu beschäftigen.

11 Helge Hveem: The political economy of Third World Producer Associations. On conditions and constraints for effective collective action among raw material producing-exporting countries. Oslo, Bergen, Tromso: Universitetsf orlaget 1977, $237 \mathrm{~S} ., 72 \mathrm{Nkr}$. 
Zur Beurteilung der Kartellfähigkeit diskutiert er folgende Indikatoren: Kohäsion der Exportländer, ihr Marktanteil, die Organisation der Konsumenteninteressen (Grad der Monopolisierung), Abhängigkeit der Produzenten von den Exporten, Elastizität der Nachfrage. Seiner Ansicht nach gibt es so im Bauxitsektor gute Möglichkeiten für Kartellaktionen (was er der existierenden IBA auch bescheinigt), etwas geringere (aber auch noch gute) im Bananen-, Phosphat-, Kautschцk- und Gewürzsektor, mittelgute Möglichkeiten immerhin noch im Kakao-, Tee- und Zuckersektor (S. 113). Hier scheint viel Wunschdenken mitzuspielen. Die politischen Probleme, die die Produzentenländer untereinander haben, um sich überhaupt auf eine gemeinsame Linie zu einigen, werden dabei zudem ganz ausgeblendet. Hveem ist jedoch zuzustimmen, daß gemeinsame bzw. konzertierte Aktionen aller Produzentenländer auf allen (oder möglichst vielen) Rohstoffmärkten deren Durchsetzungsfähigkeit erheblich verbessern würde. Dem immerhin seit 1975 in der Diskussion befindliche Rat der Produzentenvereinigungen sieht Hveem (1977!) allerdings mit zu großem Optimismus entgegen (S. $118 \mathrm{ff}$.). Er ist bis heute (1981!) - aufgrund von Finanzierungsschwierigkeiten - immer noch nicht eingerichtet worden; wie allgemein die Mithilfe der OPEC-Länder bei der Einrichtung und Stärkung von Produzentenvereinigungen sich bisher allenfalls auf ihren (kostenlosen) Vorbildcharakter und verbale Solidaritätsadressen beschränkt hat. Hveems Plädoyer ist aber sicher berechtigt, daß eine effektive Instrumentalisierung von Produzentenvereinigungen nicht nur mit kurzfristigen Erwartungen verbunden werden sollte, sondern auch in einer langfristigen Perspektive gesehen werden muß (er entwickelt ein Szenario mit 3 Phasen und 15 Jahren Dauer, S. 132/133).

Man sollte Produzentenvereinigungen der Entwicklungsländer jedoch nicht nur unter ihrer Kartelleigenschaft (d. h. ihrer Fähigkeit zur monopolistischen Beeinflussung der Weltmarktpreise) sehen und bewerten. Die größere und bessere Kontrolle der nationalen Ressourcen in Produktion und Vermarktung, der Aufbau von Linkage-Industrien können ebenso wichtige, wenn nicht sogar wichtigere und gelegentlich realistischer anzustrebende Ziele sein. Auch allein die gegenseitige Information und Marktkoordination sowie der Betrieb von Gemeinsamen Unternehmen können kleine, nichtsdestoweniger nicht unwichtige Schritte in die richtige Richtung sein. Tatsächlich haben die meisten Produzentenvereinigungen eher derart moderate Ziele und es wäre nützlich zu wissen, in welchem Umfang sie wenigstens diese zu verwirklichen in der Lage sind. Dazu würden Detailstudien der einzelnen Produzentenvereinigungen nötig sein, die allerdings, wie erwähnt, noch weithin ausstehen. ${ }^{12}$

12 Siehe dazu aber: Carlos Fortin: Die institutionelle Politik der Dritten Welt und Collective Self-Reliance: Der zwischenstaatliche Rat der kupferexportierenden Länder (CIPEC), in: K. M. Khan (Hrsg.): Self-Reliance als nationale und kollektive Entwicklungsstrategie, München 1980, S. 417-462; Rolf Hanisch: Confrontation between Primary Commodity Producers and Consumers: The Cocoa Hold-up of 1964-65, in: The Journal of Commonwealth and Comparative Politics, vol. XIII, No. 3, Nov. 1975, p. 242-260; Emil Kreider: Banana Cartel? Trends, Conditions, and Institutional Developments in the Banana Market, in: Inter-American Economic Affairs, vol. 31, 1977, No. 2, p. 3-24. 
Den relativ besten allgemeinen Uberblick bietet hier die Veröffentlichung des exil-chilenischen Okonomen Gonzalo Martner ${ }^{13}$ - Planungsminister in der Allende-Regierung der Material für diese Arbeit als Mitarbeiter des "Action Programme for Economic Cooperation among Developing Countries" der Blockfreienbewegung gesammelt hat. Bei Martner finden sich die verläßlichsten Angaben über die Entwicklung und den Stand der "Produzentenvereinigungsbewegung" (wie er sie wohl auch zu Recht charakterisiert), über Mitglieder, Ziele, Organe und (auch hier am relativ schwächsten) über die Politik. Ergänzt wird die Darstellung durch die Satzung der OPEC, des (zu gründenden) Rates der Produzentenvereinigungen, einer Modellsatzung für individuelle Produzentenvereinigungen sowie durch eine juristischen Referenzrahmen für die Zusammenarbeit zwischen Mitgliedsländern einer Produzentenvereinigung. Die Praxisorientierung des Bandes wie die relative Materialfülle machen ihn vorerst unentbehrlich für diejenigen, die am Problem der Produzentenvereinigungen von Entwicklungsländern - wo noch viele Desiderate zu schließen sind - weiter arbeiten wollen.

13 Gonzalo Martner: Producers-Exporters Associations of Developing Countries. An instrument for the establishment of a new international economic order. o. O., o. J. (Geneva 1979), 188 S. (zu beziehen von: IFDA, 2 place du Marché, 1260 Nyon, Schweiz). 